\title{
IN SITU MONITORING OF NATIVE OXIDE FILM BEHAVIOR AT MEMS CONTACT INTERFACES THROUGH BASIC ELECTRICAL MEASUREMENTS
}

\author{
L. Kogut, A. Lumbantobing, and K. Komvopoulos \\ Department of Mechanical Engineering, University of California, Berkeley, CA 94720
}

\begin{abstract}
Special surface micromachines were used to study the behavior of polysilicon/native oxide/polysilicon contact interfaces. Contact voltage measurements obtained during monotonic and cyclic contact loading were analyzed in the context of an electrical contact resistance theory in order to elucidate the contact behavior of the native oxide film. The results indicate that the oxide film remains nearly intact under monotonic contact loading, while it ruptures under cyclic contact loading, resulting in the formation of polysilicon/polysilicon asperity contacts, which decreases significantly the contact voltage for constant current across the interface. The erratic behavior of the contact voltage during cyclic contact loading is attributed to the pronounced effects of the insulating oxide film and fine oxide debris trapped at the contact interface. A reliable method to remove the native oxide film from the asperity contacts is presented, and its efficacy to change the contact interface behavior from nonohmic to ohmic is demonstrated by current versus contact voltage measurements. A good agreement was found between experimental and theoretical results for the current-contact voltage response. This study shows that the native oxide film can be removed from the contact interfaces of micromachines by electromechanical means without inducing surface damage.
\end{abstract}

\section{INTRODUCTION}

In view of the increasing number of microelectromechanical systems (MEMS) involving surface contact, understanding of the behavior of MEMS contact interfaces is imperative. Touch-mode MEMS devices are advantageous in many applications, such as electrostatic actuators [1], microswitches [2], and microrelays [3]. The performance and lifetime of such microdevices depend on the behavior of their contact interfaces. However, basic knowledge of MEMS contact interfaces is sparse due to limitations of conventional microscopy techniques to access hindered contact interfaces [4].

Polysilicon oxidizes readily upon exposure to air. When two polysilicon surfaces are brought into contact (Fig. 1) and electric current is passed through their contact interface, the flow of electrons is impeded by the constriction resistance $\left(R_{c i}\right)$ of the asperity contacts [5] and the tunnel resistance $\left(R_{t i}\right)$ of the thin insulating native oxide film separating the two surfaces [6]. Thus, the contact voltage measured across the interface can be used to characterize the contact behavior $[\mathbf{5 , 6 ]}$. Ohmic contacts are desirable in a variety of applications, such as microrelays [7]. However, the potential barrier due to the presence of an insulating film dominates the charge transport, resulting in nonohmic behavior at the contact interface.

The main objective of this study was to explore the feasibility of current and contact voltage measurements as a nondestructive in situ means of monitoring the behavior of MEMS contact interfaces

Travel support has been generously provided by the Transducers Research Foundation and by the DARPA MEMS and DARPA BioFlips programs.
To accomplish this objective, a special microdevice was designed and fabricated using surface micromachining with poly-silicon as the structural material. A simple and reliable procedure was developed to remove the native oxide film from asperity contacts and, hence, change the contact resistance behavior from nonohmic to ohmic. The efficiency of this technique was demonstrated by current versus contact voltage measurements. The MEMS devices were tested under a variety of operating conditions and the results were interpreted in the context of an electrical contact resistance (ECR) theory [6], thus providing new insight into the characteristics of MEMS contact interfaces.

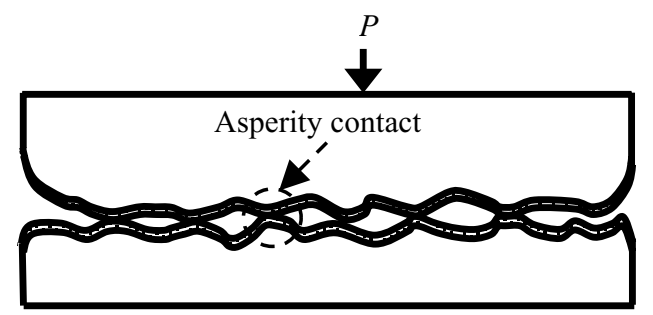

(a)

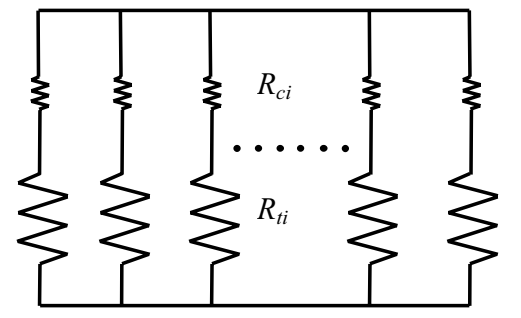

(b)

Figure 1. (a) Polysilicon surfaces in normal contact separated by an insulating native oxide film, and (b) electrical analog of the contact interface consisting of constriction resistance $R_{c i}$ and tunnel resistance $R_{t i}$ at each asperity contact.

\section{EXPERIMENTAL PROCEDURES}

Figure 2 shows a scanning electron microscope (SEM) micrograph of the MEMS device used in the present experiments. The main part of the microdevice is a microstructure (shuttle) connected to pad A. The shuttle is suspended above the substrate by a folded-flexure suspension. Four $10 \mu \mathrm{m} \times 10 \mu \mathrm{m}$ dimples at the corners of the bottom surface of the shuttle are used to control the contact area. Three electrodes below the shuttle - the center electrode (connected to pad B) and two electrically connected side electrodes (connected to pad C) that are isolated from the center electrode - are used to actuate the shuttle in the normal direction and to obtain electrical measurements. The shuttle and center electrode and the shuttle and side electrodes form an electrostatic closing gap actuator, which is used to pull the shuttle into contact with the side electrodes at the dimples. It is reasonable to postulate that the dimples share equally the contact load, and that the contact interface behavior of the four dimples and the two side electrodes is analogous to that of four identical resistors in parallel.

The test microdevice was fabricated by surface micro- 


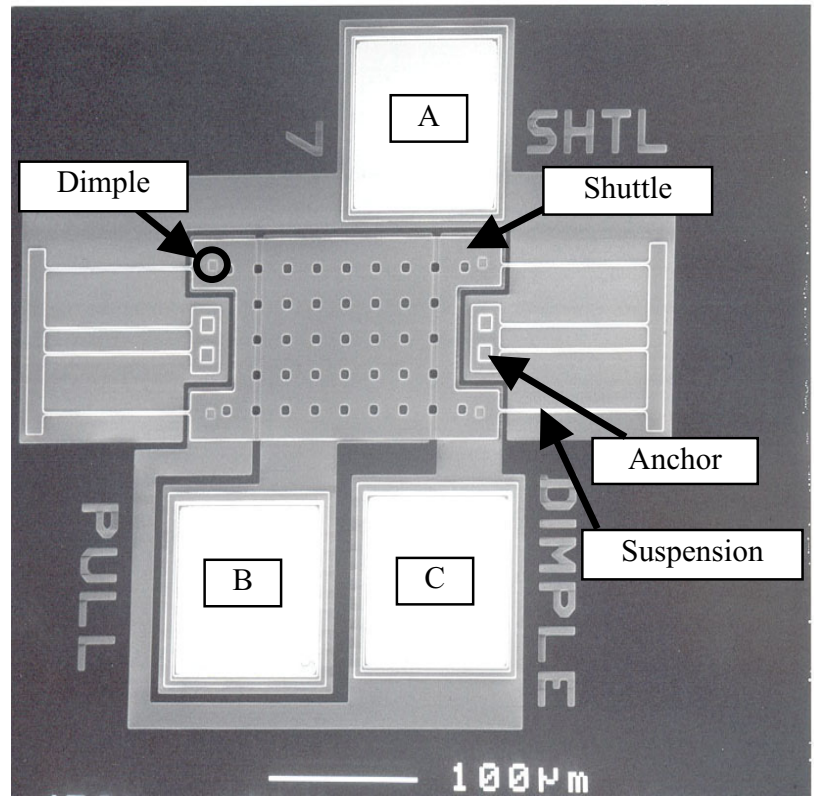

Figure 2. Scanning electron micrograph of the microdevice.

machining using multi-user MEMS processes (MUMPs). The three electrodes consist of a $0.5 \mu \mathrm{m}$ thick polysilicon layer (poly 0 ) and are isolated from the n-type $\mathrm{Si}(100)$ substrate by a $0.6 \mu \mathrm{m}$ thick $\mathrm{SiN}$ layer. The suspension system consists of a $2 \mu \mathrm{m}$ thick polysilicon layer (poly 1). The fabrication of the shuttle was completed by stacking the poly 1 and poly 2 layers (total thickness equal to $3.5 \mu \mathrm{m})$ in order to increase the rigidity of the shuttle. The 0.75 $\mu \mathrm{m}$ in height dimples at the bottom surface of the shuttle were fabricated from poly 1 . A $0.5 \mu \mathrm{m}$ thick Au layer was evaporated on the pads in order to minimize the ECR at the microprobe/pad contact interfaces. The actual dimensions of the microdevice were measured before testing to insure accurate modeling. The postfabrication lateral dimensions and film thickness of the microdevice were measured with a SEM and an optical interferometric profiler, respectively. Relatively small discrepancies (less than 10\%) were observed between actual and design dimensions. The surface topographies of the side electrodes were examined with an atomic force microscope (AFM) using a silicon tip of nominal radius of curvature equal to $10 \mathrm{~nm}$. Three 1 $\mu \mathrm{m} \times 1 \mu \mathrm{m}$ AFM images with a lateral resolution of $2.5 \mathrm{~nm}$, obtained at various locations on the electrode surfaces, were used to examine the homogeneity of the surface topography.

The experiments were performed in a high-vacuum multiprobe station (MMR Technologies, Inc., California) mounted on a vibration isolation table. A mechanical roughing pump and a turbo pump were used to evacuate the chamber to pressures as low as $10^{-6}$ Torr. The chamber, connected to a source of dry nitrogen (99.998\% purity) via a dehumidifying filter, was first pumped down to $10^{-6}$ Torr and then refilled with dry nitrogen. Unless otherwise stated, the experiments were carried out in dry nitrogen, room temperature, and atmospheric pressure.

\section{RESULTS AND DISCUSSION}

\section{Monotonic contact loading}

Measurements of contact voltage versus monotonically increasing contact pressure were obtained under constant current in order to determine the thickness of the native oxide film and to analyze its response to mechanical stresses. The shuttle was first pulled down by slowly increasing the DC bias voltage applied to the center electrode $V_{P}$ (Fig. 3). When the bias voltage reached a critical value [8], instability occurred and the shuttle snapped into contact with the substrate, forming four contact interfaces between the dimples and the side electrodes across which the contact voltage was to be measured. At that instant, the gap between the shuttle and the center electrode was equal to the height of the dimples $(0.75 \mu \mathrm{m})$. Subsequently, $V_{P}$ was increased gradually to produce a monotonically increasing contact load. At each load level, the contact voltage across the interface of the four dimples and two side electrodes was measured via a voltmeter by applying a constant current of $10 \mu \mathrm{A}$ across the interface.

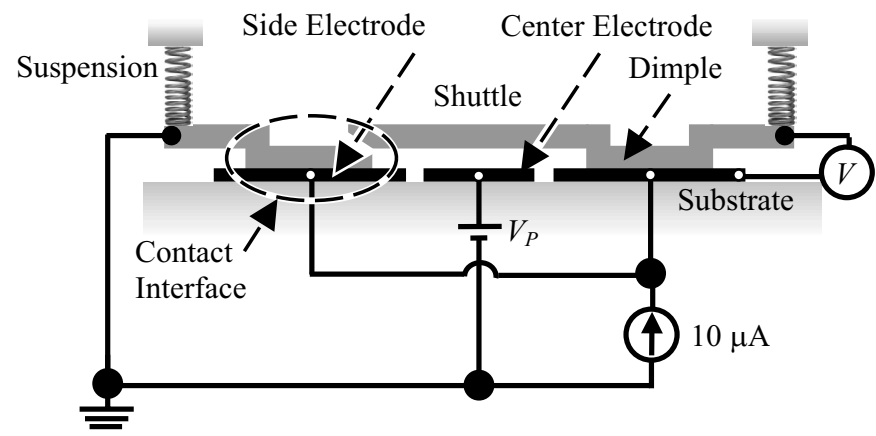

Figure 3. Schematic cross-sectional view of the microdevice showing the circuit used for contact voltage measurement in the monotonic and cyclic contact loading experiments.

Figure 4 shows the contact voltage versus apparent contact pressure $p_{a}$, defined as the ratio of the contact load to the apparent contact area. Each data point and error bar represents the average contact voltage and standard deviation, respectively, obtained from 36 microdevices selected from three different dies and tested at a given apparent contact pressure. The measured contact voltages correspond to ECR values of the order of $100 \mathrm{k} \Omega$, i.e., about five orders of magnitude higher than those obtained with MEMS devices possessing gold (ohmic) contacts $(\sim 1 \Omega)$ [9]. Since the microdevices consist of heavily doped polysilicon with specific resistivity only three orders of magnitude higher than that of gold [9], the five orders of magnitude higher ECR values shown in Fig. 4 indicate the presence of an insulating (oxide) thin film at the contact interface (nonohmic contact). The moderate decrease of the contact voltage with the increase of the apparent contact pressure is indicative of a nonohmic contact behavior. This is a characteristic of the tunnel resistance of a thin insulating film. The tunnel resistance increases with the decrease of the voltage drop across each asperity contact due to the increase of the effective barrier height $[\mathbf{1 0}]$. The increase in the apparent contact pressure produces a larger contact area and, hence, lower ECR, which, for fixed current flow $(10 \mu \mathrm{A})$, yields a smaller voltage drop across the interface. This increases the asperity contact tunnel resistances, thereby compensating the decrease of the contact voltage [6].

Polysilicon surfaces are known to readily oxidize upon exposure to room air. On average, a microdevice was exposed to the atmosphere for about $2 \mathrm{~h}$ prior to testing in dry nitrogen. Based on experimental results [11], it was predicted that a $6.5 \AA$ thick native oxide film formed on the polysilicon surfaces during the exposure to the ambient conditions. Since the thickness of the oxide film is significantly less than the measured rms surface roughness (137 $\AA$ ), it may be postulated that the oxide film did not possess a uniform thickness. Therefore, the $6.5 \AA$ value was treated as the rms value of the oxide film thickness, implying that an oxide film of effective thickness equal to $9.2 \AA$ existed at the dimple/ electrode contact interfaces. 


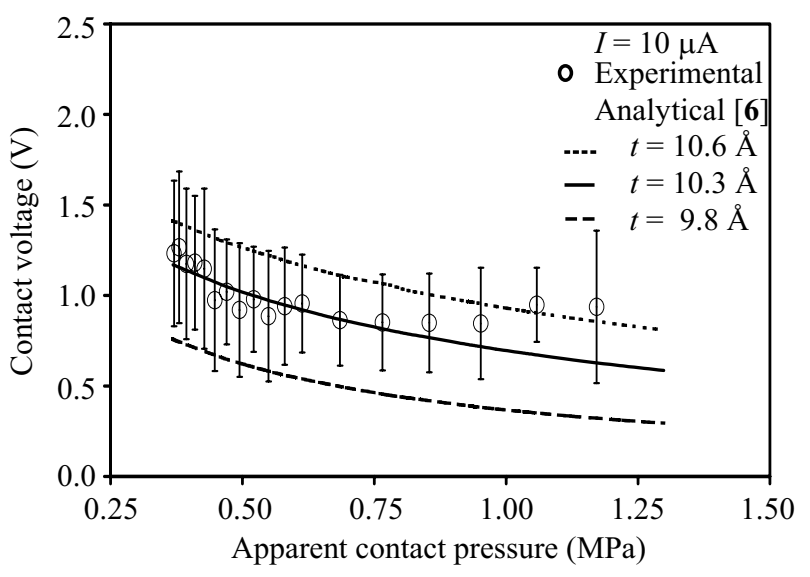

Figure 4. Contact voltage versus apparent contact pressure for monotonic contact loading.

The ECR theory reported in Ref. [6] for conductive rough (fractal) surfaces separated by a thin insulating film was used to analyze the experimental results shown in Fig. 4. In the lowpressure regime $\left(p_{a}<0.75 \mathrm{MPa}\right)$, the experimental data show a $25 \%$ decrease in average contact voltage with the increase of the apparent contact pressure. This behavior agrees well with the analytical solution obtained for insulating film thickness $t=10.3$ $\AA$. The error bars in the experimental data are bounded by solutions corresponding to $t=9.8$ and $10.6 \AA$. The $10.3 \AA$ film thickness is close to the predicted effective thickness of the native oxide film $(9.2 \AA)$. The fairly accurate theoretical predictions of the contact voltage in the low-pressure regime suggest that contact voltage measurements together with appropriate theoretical treatment can be used to determine in situ the thickness of the native oxide film at MEMS contact interfaces.

The agreement between experimental and analytical results decreases in the high-pressure regime $\left(p_{a}>0.75 \mathrm{MPa}\right)$. The measured contact voltage reaches a plateau and then increases slightly with the increase of the apparent contact pressure, contrary to the trend predicted by the analytical solutions. This is a result of the significant bending of the shuttle due to the high electrostatic force, resulting in partial surface separation of the dimples from the side electrodes. As a consequence, the contact area decreased and, in turn, the contact voltage increased (for constant current) [6]. This effect compensated the decrease of the contact voltage due to the increase of the apparent contact pressure, producing a contact voltage plateau.

\section{Cyclic contact loading}

The response of the oxide film to cyclic contact loading was examined by applying and removing a bias voltage $V_{P}$ to the center electrode (Fig. 3) in order to simulate the loading and unloading stages of a contact loading cycle. A bias voltage (maintained constant throughout a single test) was applied for $1 \mathrm{~s}$ during each loading stage. The cycle frequency was set at $0.5 \mathrm{~Hz}$, and contact voltage measurements were acquired during both loading and unloading for a constant current of $10 \mu \mathrm{A}$ applied across the contact interfaces (Fig. 3). In these tests, the electrostatic force during contact was varied between 0 and $24 \mu \mathrm{N}$ (low-load range) and 0 and $95 \mu \mathrm{N}$ (high-load range), sufficiently high to initiate contact without causing excessive bending of the shuttle, for a minimum of $10^{5}$ contact loading cycles using 10 and 8 microdevices, respectively. However, despite the removal of the electrostatic force, the shuttle was permanently adhered to the side electrodes, as indicated by the finite contact voltage measured during unloading. Stiction of the shuttle to the substrate occurred due to the relatively smaller restoring force than the adhesion force generated at the contact interface. Regardless of this limitation, valuable insight into the contact behavior was obtained from the cyclic contact loading experiments.

Figure 5 shows characteristic examples of contact voltage responses obtained during the loading stage of the cyclic contact loading experiments for electrostatic force in the high-load range. A significantly erratic behavior of the contact voltage occurred during testing. Similar results were observed for electrostatic force in the low-load range. The analytical results shown in Fig. 4 reveal that the decrease in the contact voltage can only be attributed to thinning of the insulating oxide film at asperity contacts, leading to the formation of third-body oxide debris. Using the ECR theory presented in Refs. [5] and [6], it was predicted that rupturing of the oxide film from all the asperity contacts or only from the smallest asperity contact should yield contact voltages of the order of $10^{-3}$ and $10^{-2} \mathrm{~V}$, respectively. Therefore, the contact voltage fluctuations may be attributed to redistribution of fine oxide debris produced from rupturing of the oxide film at several asperity contacts. Because only normal contact occurred, oxide debris remained within the vicinities of asperity contacts. Despite the fact that the dimples and side electrodes remained attached throughout testing, separation of several asperity contacts occurred during unloading, providing a means for oxide debris to redistribute at the contact interface and, thus, cause fluctuations in the contact voltage response.

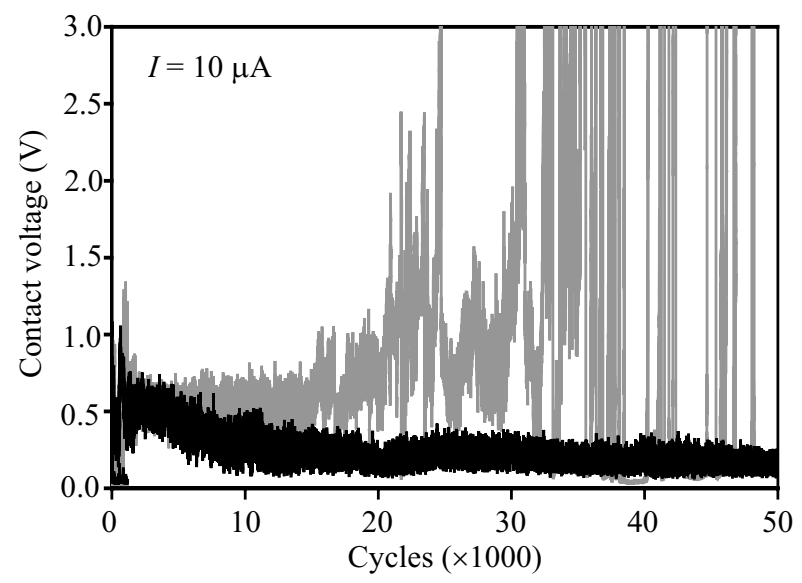

Figure 5. Examples of contact voltage responses obtained during the loading stage of cyclic contact loading experiments performed under electrostatic force varied between 0 and $95 \mu N$.

\section{Transition from nonohmic to ohmic behavior}

Since cyclic contact loading did not produce a stable ohmic response, additional experiments were performed in order to develop a reliable procedure for altering the contact behavior from nonohmic to ohmic. Figure 6 shows a schematic of the microdevice and the electrical connections used to obtain current versus contact voltage measurements. By applying a potential $V_{D}$ to the side electrodes and grounding the shuttle and the center electrodes $\left(V_{P}=0\right)$, electrostatic force was generated between the side electrodes and the free-standing shuttle. To establish contact between the shuttle and the substrate, $V_{D}$ was gradually increased until the shuttle snapped into contact with the underlying side electrodes. Once contact was established, current flow commenced between the side electrodes and the shuttle, resulting in zero electrostatic force. However, the shuttle remained stuck to the electrode surfaces, as mentioned earlier. This enabled the 
measurement of the current in terms of the contact voltage (by varying $V_{D}$ ), thereby allowing characterization of the contact interface without changing the contact load. All the results presented below were obtained from experiments conducted under working pressure of $10^{-6}$ Torr.

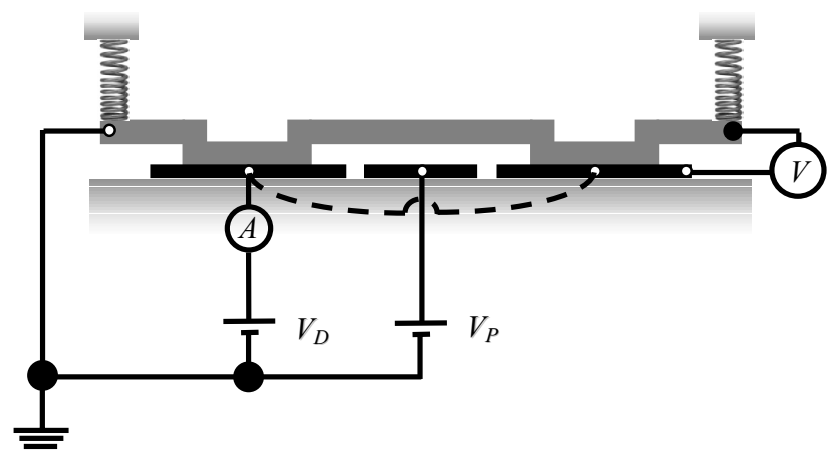

Figure 6. Schematic cross-sectional view of the microdevice showing the circuit used in the current versus contact voltage characterization.

Figure 7 shows a comparison between experimental and analytical results [6] of the current as a function of contact voltage. The experimental results were obtained from 11 microdevices selected from two different dies. For each microdevice, the current was measured for different contact voltages, while ramping $V_{D}$ up and down in the range of $0-5 \mathrm{~V}$. Solutions for the current versus contact voltage (not shown here for brevity) were obtained for different contact loads using the theory presented in Ref. [6]. A good agreement was found between experimental and theoretical results for a contact load (including adhesion) equal to $36 \mu \mathrm{N}$. Since the restoring force generated by the suspension was equal to $14 \mu \mathrm{N}$, the adhesion force [12] was estimated to be equal to $50 \mu \mathrm{N}$. This high adhesion force resulted in permanent adhesion (stiction) of the shuttle to the side electrodes. As shown in Fig. 7, the contact interface exhibited a nonohmic behavior that is attributed to the nonohmic tunnel resistance.

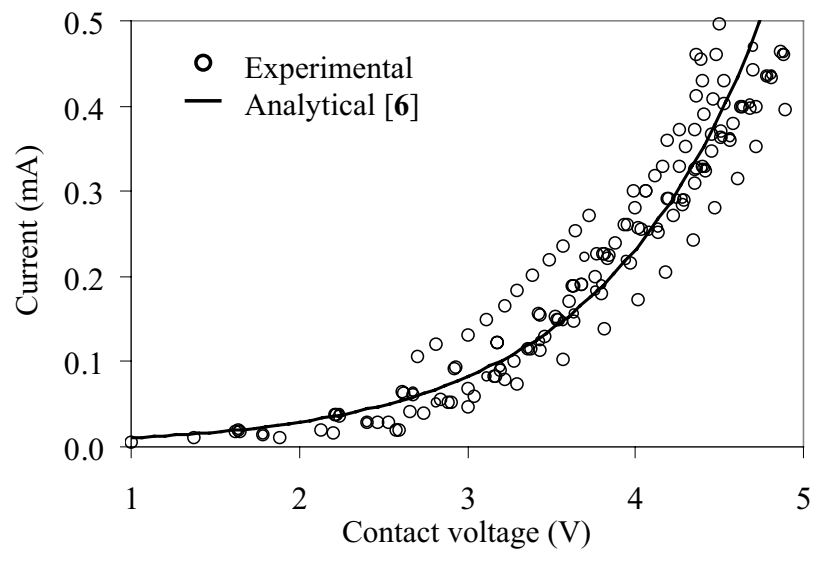

Figure 7. Current versus contact voltage after pulling the shuttle into contact showing nonohmic behavior.

Figure 8 shows that the corresponding power dissipation, obtained by multiplying the current by the contact voltage (Fig. 7), increases with the contact voltage. Since thermal softening of gold contacts occurred for similar values of power dissipation [9], it was necessary to examine whether the contact interface was altered during the measurements. Hence, instead of using the same microdevice (Fig. 4), contact voltage measurements for a given apparent contact pressure were obtained with a new microdevice. In these experiments, electrical energy about two orders of magnitude less than that in the monotonic contact loading experiments (Fig. 4) was supplied to the contact interfaces, which reduced the possibility of thermal softening. A total of 12 microdevices were used to measure the contact voltage for 12 different values of apparent contact pressure covering the entire apparent contact pressure range shown in Fig. 4. The results obtained from these experiments were found to be in good agreement with those obtained using the original testing procedure. Therefore, it is concluded that thermal softening did not occur during testing and that the variation of the contact voltage in Fig. 4 is solely due to the effect of the contact load on the real contact area.

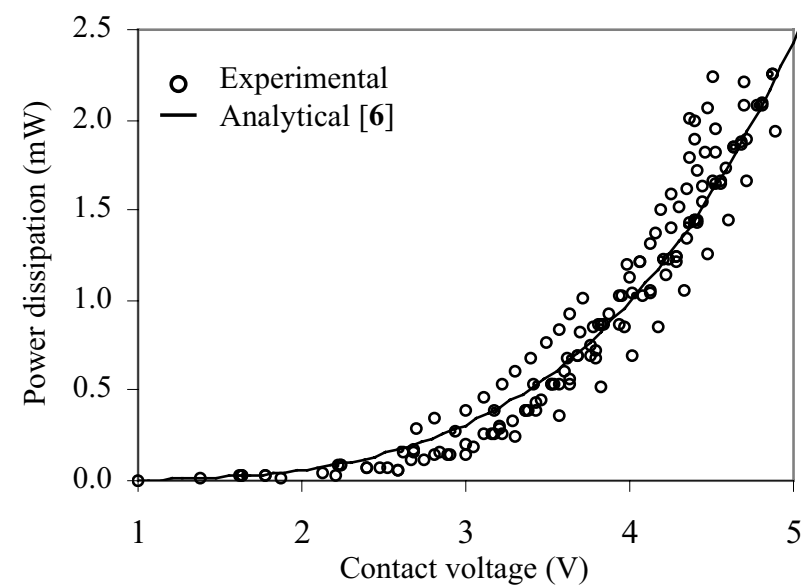

Figure 8. Power dissipation versus contact voltage response corresponding to the results shown in Fig. 7.

Figure 9 shows representative results for the current versus contact voltage behavior of a stuck microdevice (curve denoted original). The common method for releasing adhered microdevices by a mechanical microprobe was employed in these experiments. The released microdevice was pulled again into contact by increasing gradually the voltage $V_{D}$, following the procedure described earlier. The variation of the current with contact voltage $\left(1^{\text {st }}\right.$ release) shows again nonohmic behavior, suggesting that the native oxide film was not ruptured during mechanical probing. The behavior obtained after repeating this process $\left(2^{\text {nd }}\right.$ release) reveals a similar trend. Thus, the microdevice continued to exhibit nonohmic contact behavior even after it was released based on this technique. For constant contact voltage, the current decreased after each release process, presumably due to surface roughening caused by the abruptness of the probing process.

As mentioned in the previous section, applying a high electrostatic force to the shuttle by biasing the center electrode caused downward bending of the shuttle. This bending of the shuttle was examined as a means of releasing the stuck shuttle and also altering the current-contact voltage response. In these experiments, the shuttle was first pulled into contact by using the aforementioned approach based on the gradual increase of $V_{D}$. Then, the voltage $V_{P}$ applied to the center electrode (Fig. 6) was increased slowly, while the current flow corresponding to a constant voltage $V_{D}$ was monitored continuously. Only a moderate increase in the current (compared to the values obtained upon initial contact) occurred up to $V_{P}=90 \mathrm{~V}$, as discussed previously. 


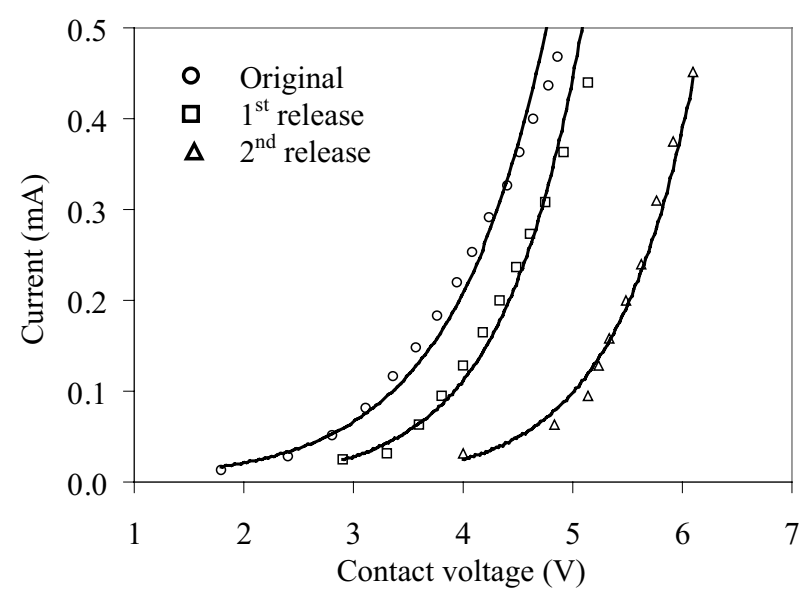

Figure 9. Current versus contact voltage for a microdevice released with a mechanical microprobe.

However, the abrupt decrease in the current observed when $V_{P}>$ $90 \mathrm{~V}$ suggested that excessive bending of the shuttle occurred, causing rocking of the dimples. As a consequence, the real contact area between the dimples and the side electrodes decreased, thus reducing the magnitude of the adhesion force at the contact interfaces. Consequently, upon the removal of the voltage $V_{P}$, the shuttle snapped back to its original free-standing position, as indicated by the current measurements (open contact).

After the first successful release using the previous method, the shuttle was pulled again into contact with the substrate by applying a voltage $V_{D}$ to the side electrodes. Figure 10 shows a linear current versus contact voltage response $\left(1^{\text {st }}\right.$ release) that reveals an ohmic behavior, indicating the removal of the oxide film from the asperity contacts. ECR simulations based on the theory presented in Ref. [6] confirmed the ohmic behavior and yielded a current-contact voltage response similar to that shown in Fig. 10 for the extreme cases where the oxide film was either absent from all the asperity contacts or only from the smallest one. The aforementioned process was repeated (e.g., $2^{\text {nd }}$ and $3^{\text {rd }}$ release in Fig. 10) resulting in a progressive increase of the current (for constant contact voltage) towards values predicted for conductive rough surfaces (ohmic contacts) [5]. The contact interface always exhibited an ohmic behavior after each release process, contrary to the erratic behavior obtained by cyclic contact loading, suggesting the permanent removal of the oxide debris from the asperity contacts comprising the real contact area. This is probably due to the bending of the shuttle that caused relative slip between the dimples and the side electrodes. Such microscopic interfacial slip resulted in the permanent removal of the oxide debris from the asperity contacts, producing a stable ohmic behavior characterized by a linear current versus contact voltage response.

Figure 11 shows the power dissipation versus contact voltage corresponding to the results shown in Fig. 10. For constant contact voltage, the power dissipation increases with the number of release cycles due to the increase of the current flow. However, for a given contact voltage, the power dissipation of microdevices with ohmic contacts assumes higher values than those with nonohmic contacts (Figs. 11 and 8, respectively) due to the higher current intensities produced (Figs. 10 and 7, respectively). The higher power dissipation may result in thermal damage of a MEMS device operating under a high contact voltage. While for ohmic contacts the power dissipation versus contact voltage is parabolic (power dissipation is proportional to the contact voltage squared and inversely proportional to the contact resistance, Fig. 11), for

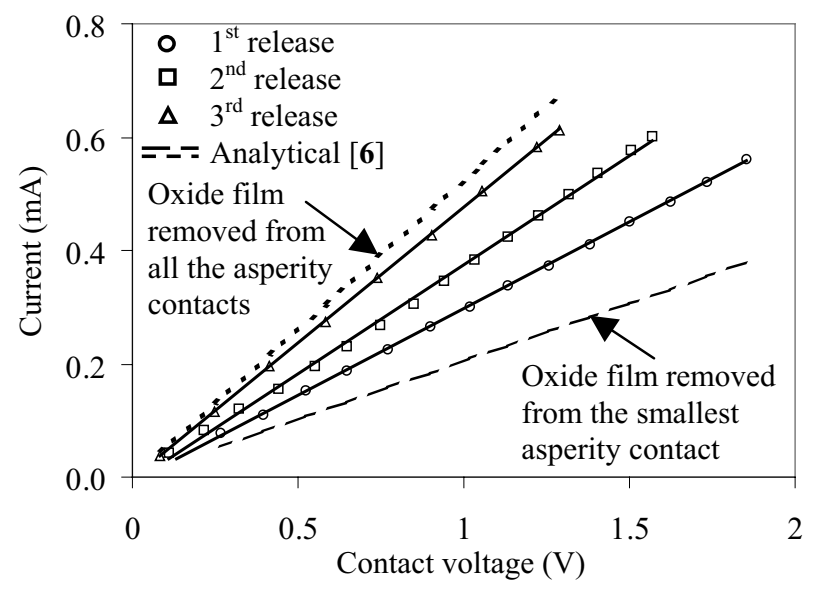

Figure 10. Current versus contact voltage for a microdevice released by applying and removing a relatively high electrostatic force to cause bending and snapping back of the shuttle. The analytical results were obtained from the theory presented in Ref. [6] assuming that the native oxide film was removed from the smallest asperity contact and all the asperity contacts.

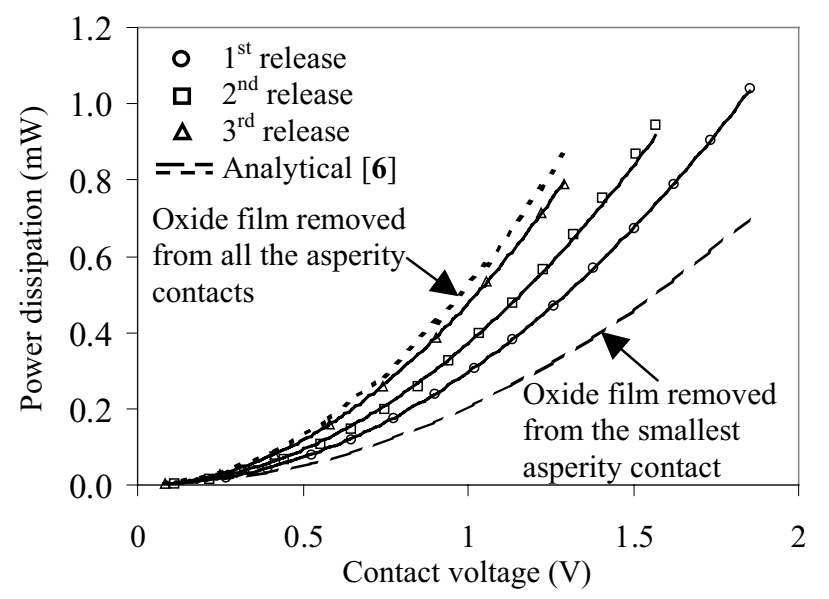

Figure 11. Power dissipation versus contact voltage responses corresponding to the results shown in Fig. 10.

nonohmic contacts, the contact resistance depends on the contact voltage and, therefore, the relationship of the power dissipation and the contact voltage is not parabolic (Fig. 8).

Figure 12 shows the variation of the current with time when the stage temperature was increased from $35^{\circ} \mathrm{C}$ to $155{ }^{\circ} \mathrm{C}$ in four steps of $2 \mathrm{~min}$ each. In the first minute of each cycle, the stage temperature increased linearly at a rate of $30{ }^{\circ} \mathrm{C} / \mathrm{min}$. The temperature was maintained constant during the second minute of each step. As shown in Fig. 12, the current response lagged the temperature change by a few seconds (typically 5 to 8 seconds) and the current decreased with the increase of the stage temperature. The time lag is probably a result of the thermal resistance of the microdevice-stage interface and the contact interfaces between the polysilicon surfaces. The change in the current is relatively small (less than $10 \%$ of the original value for a temperature change of $120^{\circ} \mathrm{C}$ ). However, the current variation of $\sim 1 \mathrm{~mA}$ can easily be resolved with a standard ampermeter of resolution equal to $1 \mu \mathrm{A}$, corresponding to a temperature resolution of $0.12{ }^{\circ} \mathrm{C}$ for a dynamic range of $120{ }^{\circ} \mathrm{C}$. This demonstrates the possibility of using current measurements in MEMS devices possessing contact interfaces as a means of in situ 
surface temperature sensing. The small temperature effect on the current is attributed to the fact that the tunnel resistance is practically independent of temperature [13], and the constriction resistance, which increases with the increase of the temperature, is much smaller than the tunnel resistance [6]. Therefore, for increased sensitivity, devices with ohmic contacts are more advantageous.

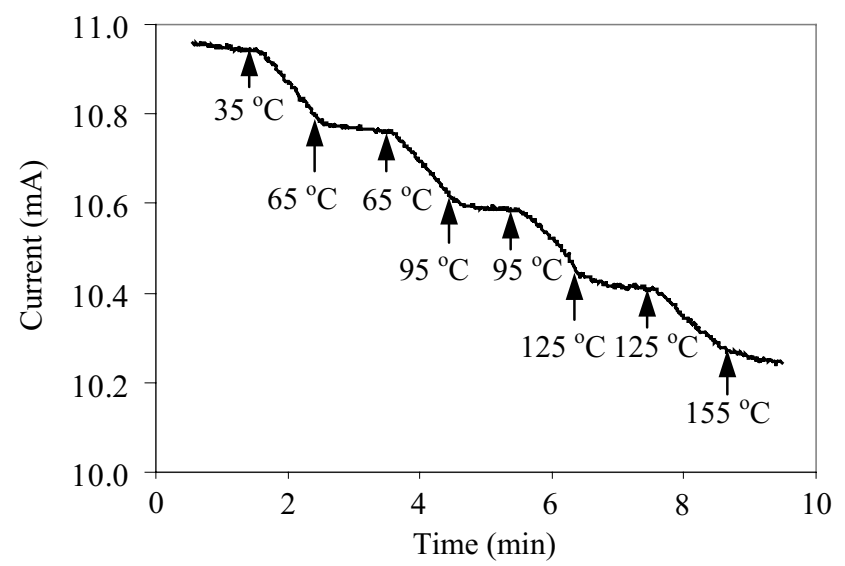

Figure 12. Current versus time showing the thermal response at the contact interface. The temperatures shown are stage temperatures, and the heating rate was equal to $30^{\circ} \mathrm{C} / \mathrm{min}$.

The present work can be extended to analyze in situ the deformation behavior of an insulating film at contacts of microswitches and microrelays. The approach derived in this study for removing the native oxide film, which is responsible for the nonohmic behavior of MEMS contact interfaces, is straightforward and can easily be adopted in various microdevices to improve the reliability and operation performance.

\section{CONCLUSIONS}

The design of a polysilicon microdevice fabricated by surface micromachining, suitable for characterizing the native oxide film at MEMS contact interfaces through basic electrical measurements, was demonstrated in this study. The electrical response of the contact interface was interpreted in terms of an earlier ECR theory to provide insight into the behavior of the oxide film under various contact loadings. Based on the presented results and discussion the following main conclusions can be drawn from this investigation.

(1) Under monotonic contact loading, the voltage decreases with the increase of the apparent contact pressure due to the increase of the real contact area. The measured voltage is less sensitive to changes in the contact pressure than ohmic contacts due to the intrinsic behavior of nonohmic contacts.

(2) The thickness of the oxide film extracted from voltage versus contact pressure measurements and an earlier ECR theory was found to be equal to $\sim 10 \AA$. The fact that the oxide thickness was not affected by contact loading is indicative of the durability of the oxide film under monotonically increasing contact loads.

(3) Cyclic contact loading promoted rupturing of the oxide film, resulting in polysilicon/polysilicon asperity contacts that reduced the contact voltage significantly. However, redistribution of oxide debris at asperity contacts produced erratic contact voltage response.

(4) The removal of the oxide film from asperity contacts through the application of electrostatic forces resulted in the release of the initially adhered microdevice and the transition from nonohmic to ohmic contact behavior.

(5) The current decreased with the increase of the temperature at the contact interface in a characteristic manner, illustrating the potential for in situ measurement of temperature changes at MEMS contact interfaces.

\section{ACKNOWLEDGEMENTS}

This research was supported by the National Institute of Standards and Technology under Grant No. 60NANB1D0078. The authors are grateful to S. J. Timpe for assistance in redesigning the MEMS devices.

\section{REFERENCES}

1. C. Cabus, E. I. Cabuz, T. R. Ohnstein, and R. Maboudian, "Factors Enhancing the Reliability of Touch-Mode Electrostatic Actuators," Sens. Actuators A, 79, 245 (2000).

2. S. Majumder, N. E. McGruer, G. G. Adams, P. M. Zavracky, R. H., Morrison, and J. Krim, "Study of Contacts in an Electrostatically Actuated Microswitch," Sens. Actuators A, 93, 19 (2001).

3. H.-S. Lee, C. H. Leung, J. Shi, S.-C. Chang, S. Lorincz, and I. Nedelescu, "Integrated Microrelays: Concept and Initial Results", J. Microelectromech. Syst., 11, 147 (2002).

4. I. De Wolf and W. M. van Spengen, "Techniques to Study the Reliability of Metal RF MEMS Capacitive Switches," Microelectron. Reliab., 42, 1789 (2002).

5. L. Kogut and K. Komvopoulos, "Electrical Contact Resistance Theory for Conductive Rough Surfaces," J. Appl. Phys., 94, 3153 (2003).

6. L. Kogut and K. Komvopoulos, "Electrical Contact Resistance Theory for Conductive Rough Surfaces Separated by a Thin Insulating Film,” J. Appl. Phys., 95, 576 (2004).

7. I. Schiele and B. Hillerich, "Comparison of Lateral and Vertical Switches for Application as Microrelays," J. Micromech. Microeng., 9, 146 (1999).

8. M.-H. Bao, Handbook of Sensors and Actuators, vol. 8: Micro Mechanical Transducers, Pressure Sensors, Accelerometers, and Gyroscopes, S. Middelhoek, Ed., New York: Elsevier, 2000, pp. 140-142 and 144-150.

9. E. J. J. Kruglick and K. S. J. Pister, "Lateral MEMS Microcontact Considerations,” J. Microelectromech. Syst., 8, 264 (1999).

10. J. G. Simmons, "Generalized Formula for the Electric Tunnel Effect Between Similar Electrodes Separated by a Thin Insulating Film," J. Appl. Phys., 34, 1793 (1963).

11. W. B. Ying, Y. Mizokawa, Y. Kamiura, K. Kawamoto, and W. Y. Yang, "The Chemical Composition Changes of Silicon and Phosphorous in the Process of Native Oxide Formation of Heavily Phosphorous Doped Silicon,” Appl. Surf. Sci., 181, 1 (2001).

12. L. Kogut and K. Komvopoulos, "Analysis of Interfacial Adhesion Based on Electrical Contact Resistance Measurements," J. Appl. Phys., 94, 6386 (2003).

13. J. G. Simmons, "Electric-Tunnel Effect and its Use in Determining Properties of Surface Oxides," Trans. Metall. Soc. AIME., 233, 485 (1965). 